\title{
Ultrasonic protein crystallization: Promoting nucleation in microdroplets through pulsed sonication
}

\author{
Joana Ferreira,${ }^{1,2}$ Jeroen Opsteyn, ${ }^{1}$ Fernando Rocha, ${ }^{2}$ Filipa Castro ${ }^{2}$ and Simon Kuhn ${ }^{1}$
}

${ }^{1}$ KU Leuven, Department of Chemical Engineering, Celestijnenlaan 200F, 3001 Leuven,

Belgium

${ }^{2}$ LEPABE - Laboratory for Process Engineering, Biotechnology and Energy, Faculty of Engineering, University of Porto, Rua Dr. Roberto Frias, 4200-465 Porto, Portugal

\section{Keywords}

Protein crystallization, nucleation, pulsed ultrasound

\section{Highlights}

○ Promoting protein nucleation in microdroplets through low-frequency pulsed sonication

- Parametric study supported by literature to quantify the influence of the applied ultrasound energy on the induction time in an extended range of supersaturation ratios

- Controlling nucleation rate by means of alternating the supersaturation ratio and sonication pulse time

- Protein crystal production with a uniform size distribution without causing precipitation 


\begin{abstract}
Droplet microfluidics allows a higher degree of control over the crystallization conditions than conventional methodologies. To extend this approach, this work explores the synergistic effect of low-frequency pulsed ultrasound on lysozyme crystallization in microdroplets. Pulsed actuation allows control of the crystallization temperature, while the ultrasound effect significantly reduces the induction time and crystal size. Therefore, protein nucleation is enhanced by pulsed sonication without causing precipitation, resulting in uniform crystal size. Finally, the initial supersaturation ratio has a crucial contribution to the crystal size for silent experiments, while a threshold for induction time is observed in ultrasonic crystallization.
\end{abstract}

\title{
Introduction
}

The complexity of crystallizing biological macromolecules stands out compared to other molecules (i.e. organic and inorganic compounds), not only due to the stochastic nature of nucleation, where high supersaturation ratios are required (García-Ruiz, 2003), but mainly due to the dynamic behaviour of proteins and slow nucleation and crystal growth kinetics (Galkin and Vekilov, 1999). For this reason, it becomes imperative to develop more systematic crystallization strategies, since it is often a matter of trial-and-error to successfully crystallize proteins (Dombrowski et al., 2010; Gerdts et al., 2006; Revalor et al., 2010). Droplet microfluidics, where the crystals are confined inside microdroplets dispersed in an immiscible liquid, represents an attractive tool for high-throughput screening experimentation (Lau et al., 2007; Selimović et al., 2010; Zheng et al., 2003) as the transport phenomena are intensified, mostly due to the large surface-to-volume ratio (Anna, 2016; Günther and Jensen, 2006; Seemann et al., 2012).

Protein crystallization is also highly affected by external fields, e.g. electric and magnetic fields, and light and ultrasound irradiation (Revalor et al., 2010), potentially acting as nucleation 
promoters. Electric fields tend to narrow the spatial location of the nucleation events through the alignment of the molecules. This results in an increase in crystal quality and yield, with the combined reduction of induction time and crystal number (Alexander and Radacsi, 2019; Li and Lakerveld, 2018; Revalor et al., 2010; Taleb et al., 1999). Additionally, Rodríguez-Romero and co-workers (Rodríguez-Romero et al., 2017) reported that high-quality single protein crystals are obtained under an electric pulse-wave mode. Magnetic fields do not show a clear effect on nucleation, only on the crystal orientation (Astier et al., 1998; Revalor et al., 2010; Yan et al., 2016) and quality (Yin, 2015), while light irradiation induces nucleation by forming radicals (Okutsu, 2007; Revalor et al., 2010; Veesler et al., 2006). Finally, ultrasound irradiation increases the nucleation rate (Nanev and Penkova, 2001) and, consequently, reduces the induction time and the metastable zone width (Crespo et al., 2010; Kakinouchi et al., 2006; Kitayama et al., 2013; Mao et al., 2020; Revalor et al., 2010). Moreover, the production of uniform tiny crystals might result in these dispersed crystals acting as seeds for industrial macromolecular crystallization processes (Mao et al., 2020). Zhang and co-workers (2015) (Zhang et al., 2015), Ruecroft and coworkers (2015) (Ruecroft et al., 2005) and Ratsimba and co-workers (1999) (Ratsimba et al., 1999) reviewed the new perspectives and developments in terms of the industrial application of sonocrystallization. However, the crystallization of macromolecules requires accurate control of process parameters (i.e. $\mathrm{pH}$ and temperature) to avoid protein denaturation or precipitation (Mullin, 2001).

Kakinouchi and co-workers (Kakinouchi et al., 2006) performed microbatch crystallization experiments in microwells with a volume of $70 \mu 1$ by applying two short pulses (10 s and $1 \mathrm{~min}$ ) of $100 \mathrm{kHz}$ and $100 \mathrm{~W}$. The results point out a clear increase of the nucleation rate already after the first pulse, while long-term irradiation results in cluster damage without the generation of any nuclei (Kakinouchi et al., 2006). Crespo and co-workers (Crespo et al., 2010) validated the theoretical prediction of ultrasound acting as a nucleation promoter via reducing the metastable zone width. This study was conducted through vapour diffusion experiments with droplet volumes of $8 \mu 1$. An ultrasound signal of $37 \mathrm{kHz}$ and $80 \mathrm{~W}$ within pulses of continuously 
alternated sonication periods ( $3 \mathrm{~s}$ ) was established, followed by silent periods of $3 \mathrm{~min}$. The results indicate that ultrasound leads to crystal growth at lower supersaturation ratios and, consequently, crystals with better diffraction properties. Cao and co-workers (Cao et al., 2012) were able to generate large protein crystals in acoustically levitated droplets (frequency of $20 \mathrm{kHz}$ and maximum forward power of $30.5 \mathrm{~W}$ ) up to volumes of around $100 \mu 1$. Due to the absence of inertial effects, the obtained crystals exhibit better diffraction quality and less lattice imperfections. Kitayama and co-workers (Kitayama et al., 2013) performed batch experiments in a volume of $2 \mathrm{ml}$ at a frequency of $18 \mathrm{kHz}$. With a continuous cycle of alternated sonication and silent conditions (sonication for $1 \mathrm{~min}$ followed by a silent time of $2 \mathrm{~min}$ ), the acceleration of nucleation kinetics was verified. Furthermore, in the unsonicated condition the formation of larger crystals occurs due to the suppression of new nucleation events (Kitayama et al., 2013). More recently, Mao and co-workers (Mao et al., 2020) conducted an extended study of the influence of pulsed sonication during nucleation and crystal growth on the induction time, metastable zone width, crystal size, morphology, and process yield. The batch (under magnetic stirring) experiments performed in a volume of $30 \mathrm{ml}$ showed that ultrasound irradiation leads to shorter induction times. Pulsed sonication during the crystal growth stage results in smaller crystal size. Additionally, ultrasonic crystallization in segmented flow has been only explored for organic molecules, which includes the works of Rossi and co-workers (2015) (Rossi et al., 2015) on the continuous sonocrystallization of adipic acid in droplet flow, Jiang and co-workers (2015) (Jiang et al., 2015) on the continuous sonocrystallization of L-asparagine monohydrate in gas-slurry flow, and Eder and co-workers (2012) (Eder et al., 2012) on the continuous sonocrystallization of acetylsalicylic acid in gas-slurry flow.

Nucleation is a stochastic and thermally activated process with a certain activation energy (García-Ruiz, 2003). Ultrasound is hypothesized to be a nucleation promoter (Zhang et al., 2015) because of a theoretical decrease in the energy barrier for nuclei formation, due to cavitation and microstreaming (Nagy and Braatz, 2012). However, the influence of the ultrasonic power and sonication pulse time on protein crystals confined in microdroplets has, at least to our 
best knowledge, so far not been studied. This is done through the crystallization of a model protein (i.e. lysozyme) as a proof-of-concept. Initially, the effect of the forward power on the experimentally measured parameters (crystals size and number, and induction time) is investigated. Based on this, the nucleation rate after $20 \mathrm{~h}$ at several forward powers is estimated. Furthermore, the sonication pulse time is increased, and the crystallization outcome is analysed. Finally, a comparative study with the reported literature is conducted and the influence of the total ultrasound energy on the induction time at different initial supersaturation ratios is analysed.

\section{Materials and Methods}

\section{Experimental setup}

The experimental setup to achieve ultrasound activated protein crystallization is depicted in Figure 1, while the experimental methodology is described by Ferreira and coworkers (Ferreira et al., 2018). The microbatch crystallization experiments were conducted in microdroplets with a volume equal to $0.1 \mu 1$ (droplet length, $L_{\mathrm{d}}$ ), generated in a microchannel with a characteristic dimension $\left(d_{\mathrm{t}}\right)$ of $500 \mu \mathrm{m}$ (stable squeezing regime), and flow rates of the continuous (CP) and the dispersed (DP) phases equal to $0.4 \mathrm{ml} \cdot \mathrm{min}^{-1}$ and $0.3 \mathrm{ml} \cdot \mathrm{min}^{-1}$, respectively. 
(a)

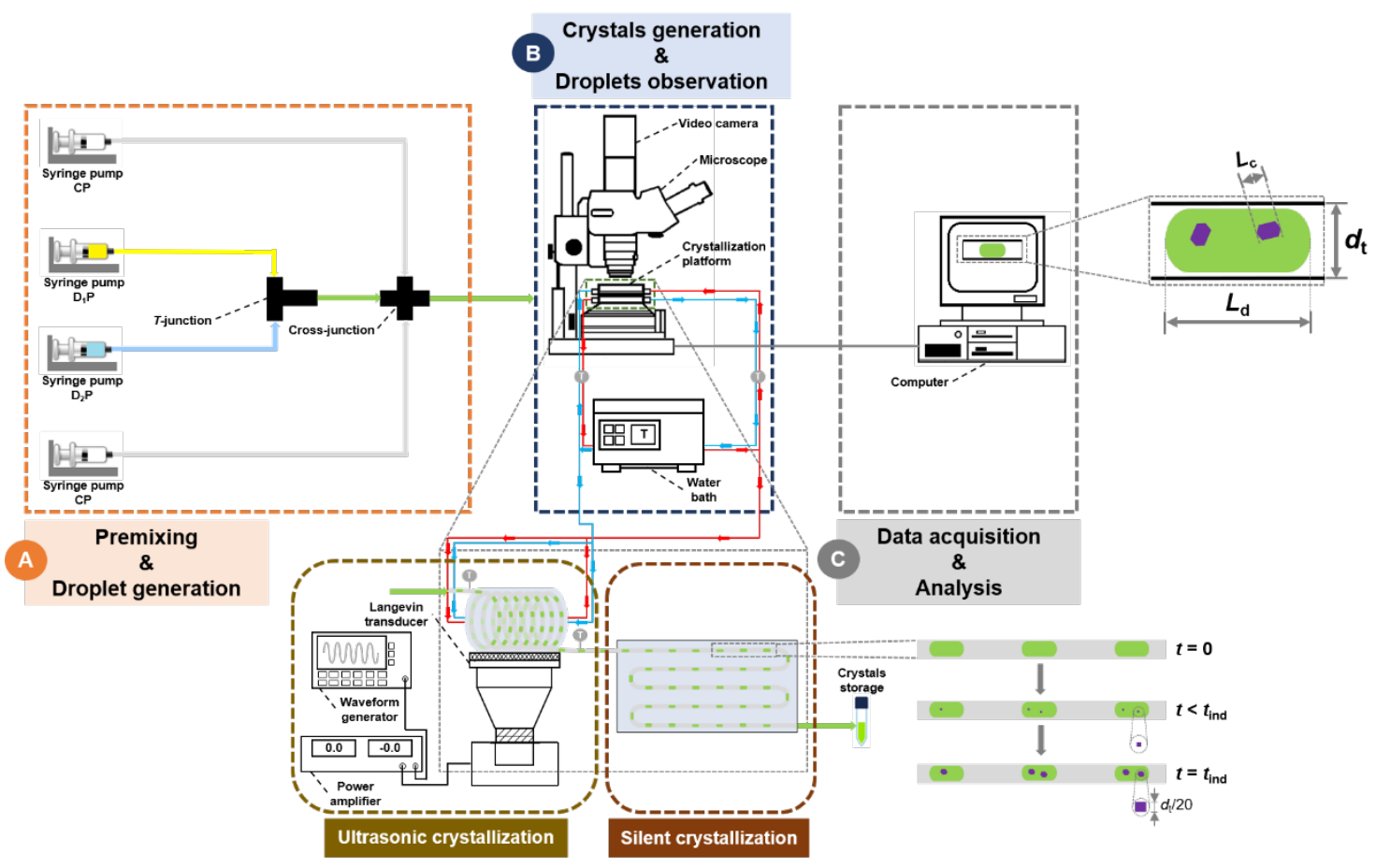

(b)

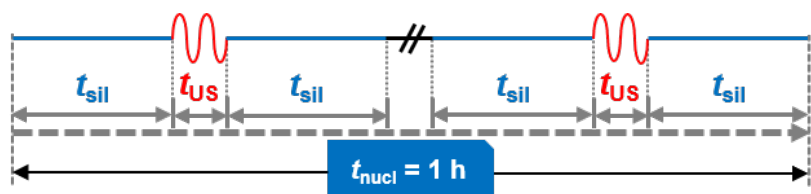

Figure 1. (a) Schematic representation of the experimental set-up: (A) Injection of the fluid phases using syringe pumps at room temperature; (B) Crystallization platform with an integrated temperature controller, which includes ultrasonic and silent crystallizations. Optical microscopy is used to monitor the experiments and the crystals are collected at the end of the platform; and (C) Crystal counting, and droplet length and crystal size measurements by image analysis techniques. (b) Schematic representation of pulsed sonication cycles.

In Section (A), the crystallization solutions (lysozyme and salt solutions) are premixed, while the droplets are generated after adding the continuous phase (silicone oil) to the system (Ferreira et al., 2018). The crystallization platform in Section (B) includes a coiled PFA tubing, which is sonicated during the ultrasound experiments (Ultrasonic crystallization section) as well as temperature control by fluid circulation through a thermostatic bath (Huber Ministat 230). The inlet and outlet temperatures are measured by thermocouple elements (Omega HH374). This coiled PFA tubing (tube-in-tube heat exchanger) is glued to a Langevin-type transducer, which is operated at its resonance frequency of $40.1 \mathrm{kHz}$, supplied by a waveform generator (Keysight 
$33500 B$, series waveform), and amplified by a power amplifier (EI, RF $2100 \mathrm{~L}, 100 \mathrm{~W})$. The output signal is checked by an oscilloscope (Keysight DSOX112A, Digital Storage Oscilloscope, $70 \mathrm{MHz}-2 \mathrm{GSa} \cdot \mathrm{s}^{-1}$ ) (Delacour et al., 2019; Dong et al., 2020, 2019). Normally, a uniform distribution of the pulse intensity for the droplets in the Ultrasonic crystallization section is observed without any preferential distribution. Besides the coiled PFA tubing, Section (B) also includes a microreactor device [Silent crystallization section in Figure 1-(a)], which is also temperature controlled.

After the generation of stable droplets, flow is stopped, and the sonication starts up to a forward power value of $53 \mathrm{~W} \cdot$ cycle $^{-1}$. The term forward power refers to the difference between the applied and reflected electric power of the amplifier. This is done following a burst sonication (pulsed ultrasound) profile as represented in Figure 1-(b). During $1 \mathrm{~h}$ (imposed nucleation time, $\left.t_{\text {nucl }}\right)$, generated droplets in the Ultrasonic crystallization section were subjected to ultrasonic radiation for an interval of $3 \mathrm{~s} \cdot \mathrm{cycle}^{-1}$ or $10 \mathrm{~s} \cdot \mathrm{cycle}^{-1}\left(t_{\mathrm{US}}\right)$, followed by an interval of $3 \mathrm{~min}$ without radiation $\left(t_{\text {sil }}\right)$. This cycle is repeated until the imposed nucleation time is reached. The total ultrasound energy $\left(E_{\mathrm{tot}}\right)$ during the irradiation time reaches values up to $10.6 \mathrm{~kJ}$. All the droplets and crystals are analysed in Section (C) after a crystallization time of $20 \mathrm{~h}$ and, consequently, the experimental images shown in the Results and Discussion section represent the outcome of three independent experiments after that time. Furthermore, isothermal experiments were conducted under three different initial supersaturation ratios $\left(S_{0}\right)$, defined as the ratio between the initial protein concentration in solution $\left(C_{0}\right)\left[\mathrm{kg} \cdot \mathrm{m}^{-3}\right]$ and the protein solubility $\left(C_{\mathrm{s}}\right)\left[\mathrm{kg} \cdot \mathrm{m}^{-3}\right]$ (Ducruix and Giegé, 1999), at pH 4.7 by changing the initial lysozyme concentration and temperature, as shown in Figure 2 and Table 1 (Forsythe et al., 1999). The initial sodium chloride concentration was $3 \%(\mathrm{w} / \mathrm{v})$. These values were selected based on a previous derived phase diagram (Ferreira et al., 2018). As described by Ferreira and co-workers (Ferreira et al., 2018), the lysozyme (chicken egg-white) used in this work is commercially supplied as a lyophilized powder. The lysozyme preparation is ensured by the supplier, which involves an initial purification, followed by a crystallization step performed three times, and dialysis. All lysozyme solutions are freshly 
prepared, without any external agitation and waiting $6 \mathrm{~h}$ before starting the experiment to ensure lysozyme dissolution.

Table 1. Initial supersaturation ratios and crystallization conditions for the performed sonocrystallization experiments (Forsythe et al., 1999).

\begin{tabular}{ccc}
\hline $\begin{array}{c}\text { Initial supersaturation } \\
\text { ratio, } \mathbf{S}_{\mathbf{0}}[-]\end{array}$ & $\begin{array}{c}\text { Crystallization } \\
\text { temperature, } \boldsymbol{T}\left[{ }^{\circ} \mathbf{C}\right]\end{array}$ & $\begin{array}{c}\text { Initial lysozyme } \\
\text { concentration, } \boldsymbol{C}_{\mathbf{0}}\left[\mathbf{m g} \cdot \mathbf{m l}^{-1}\right]\end{array}$ \\
\hline 7.2 & 15 & 30 \\
\hline 4.2 & 24 & 45 \\
\hline 3.8 & 25 & 45 \\
\hline
\end{tabular}

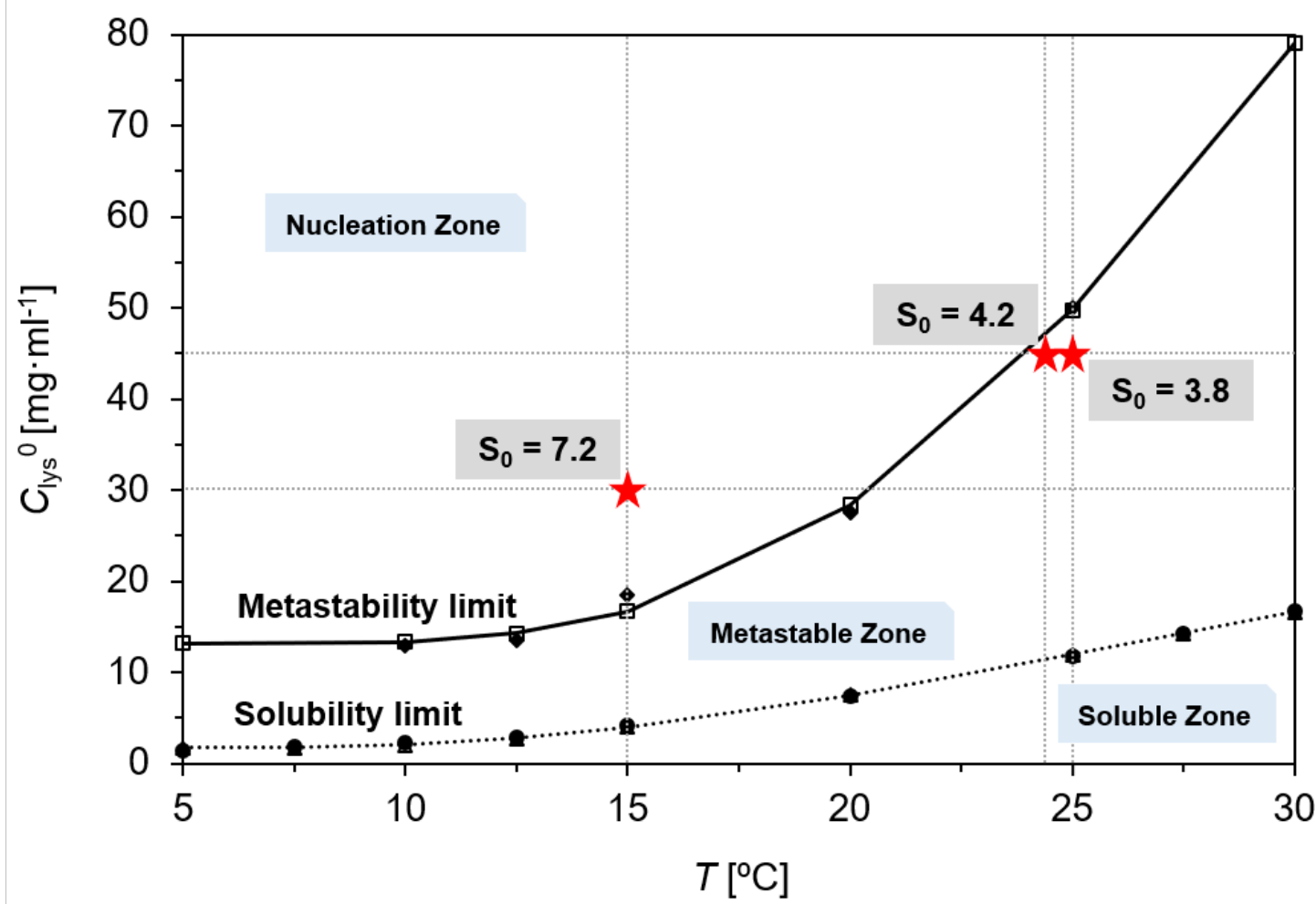

Figure 2. Lysozyme phase diagram for a droplet volume of $0.9 \mu 1$ at sodium chloride $3 \%(\mathrm{w} / \mathrm{v})$ and $\mathrm{pH} 4.7$ with the indication of the three studied initial supersaturations $\left(\mathrm{S}_{0}\right)$. [The exponential fits were obtained using van't Hoff equation (solubility limit) and Markov approximation (metastability limit), and the experimental points are reported by (Ferreira et al., 2018)].

\section{Induction time and nucleation rate}

The droplet analysis is done using NIS-Elements Advanced Research (B.V., 2019) and ImageJ software (“ImageJ," 2019). The crystal number is counted, while the size is determined 
by the equivalent diameter of a circle with an equal surface area as the projected crystal surface area. On average, 250 droplets per experiment are analysed, and three independent experiments are conducted. The induction time is determined as the first moment an observable crystal appears in a single droplet, using time-lapse capturing. For this, the crystallization platform is placed under the microscope, and a picture is taken every minute (Figure 1). A threshold corresponding to the area of a square with width equal to $d_{\mathrm{t}} / 20$ is chosen, which corresponds to a circle with identical area and a diameter of $d_{\mathrm{t}} / \sqrt{100 \pi}\left(d_{\mathrm{t}}=500 \mu \mathrm{m}\right)$. This threshold value ensures that the first moment of an observable crystals is detected with the current visualization setup. Once the crystal exceeds this threshold value, the measured time is set as the induction time. Following this, the induction time $\left(t_{\text {ind }}\right)[\mathrm{s}]$ corresponds to the time between the moment of supersaturation and the first detection of crystals (Maeki et al., 2014). As an initial approximation, it can be calculated by (Maeki et al., 2014)

$$
t_{\text {ind }}=t_{\mathrm{ss}}+t_{\mathrm{n}}+t_{\mathrm{g}}
$$

where $t_{\mathrm{ss}}[\mathrm{s}]$ is the time to reach a quasi-steady-state distribution of clusters, $t_{\mathrm{n}}$ [s] the time required to form a nuclei of critical size (nucleation time), and $t_{\mathrm{g}}[\mathrm{s}]$ the growing period of a nuclei until it reaches a detectable size. Simplifying, the theoretical induction time takes only into account two contributions from Equation (1) and the new empirical correlation is (DuránOlivencia and Otálora, 2013)

$$
t_{\text {ind }}=\frac{k_{\mathrm{t}}}{J_{\mathrm{n}} V_{\mathrm{d}}}+t_{\mathrm{g}}
$$

where $J_{\mathrm{n}}$ [\# of nuclei $\mu \mathrm{l}^{-1} \cdot \mathrm{s}^{-1}$ ] is the homogeneous nucleation rate (number of nuclei per unit volume and per unit time that exceeds the critical size), $V_{\mathrm{d}}[\mu 1]$ the droplet volume, and $k_{\mathrm{t}}[-]$ a fitting parameter. With the nucleation rate values estimated experimentally, $k_{\mathrm{t}}$ and $t_{\mathrm{g}}$ can be obtained from the fitting of Equation (2) through the minimization of the sum of quadratic errors between the theoretically calculated and experimentally obtained induction times 
$\left[\sum\left(t_{\text {ind,exp }}-t_{\text {ind,analyt }}\right)^{2}\right]$. This quadratic error for $\mathrm{S}_{0}=7.2$ results in $0.032 \mathrm{~s}^{2}$. The values of $k_{\mathrm{t}}$ and $t_{\mathrm{g}}$ are 150.1 and $1.7 \mathrm{~h}$, respectively.

The homogeneous nucleation rate can be calculated based on the average number of crystals per droplet $\left(\overline{\mathrm{N}_{\mathrm{c}}}\right)$, where this last one is determined by (Dombrowski et al., 2010; Heymann et al., 2014)

$$
\overline{\mathrm{N}_{\mathrm{c}}}=\mathrm{N}_{\mathrm{c}}^{\infty}\left(1-e^{-k_{\mathrm{nc}} t}\right)
$$

where $\mathrm{N}_{\mathrm{c}}^{\infty}$ is the number of crystals after infinite time (at the end of the crystallization experiment), and $k_{\mathrm{nc}}\left[\mathrm{s}^{-1}\right]$ a proportionality constant. Finally, the nucleation rate is estimated by (Dombrowski et al., 2010; Heymann et al., 2014)

$$
\mathrm{N}_{\mathrm{c}}^{\infty}=V_{\mathrm{d}}\left(\frac{J_{\mathrm{n}}}{D_{\mathrm{p}}}\right)^{3 / 5},
$$

where $D_{\mathrm{p}}\left[\mathrm{m}^{2} \cdot \mathrm{s}^{-1}\right]$ is the diffusion coefficient of the protein $\left[1.4 \times 10^{-10} \mathrm{~m}^{2} \cdot \mathrm{s}^{-1}\right.$ for lysozyme at $20{ }^{\circ} \mathrm{C}$ (Howard et al., 2009)].

\section{Results and Discussion}

\section{Effect of the forward ultrasound power on the crystallization outcome}

The influence of the forward power on the crystallization experiments performed at $\mathrm{S}_{0}=7.2$ is shown in Figures 3 and 4 , and Table 2. Figure 3 presents the lysozyme crystals generated in the confined microdroplets for silent conditions and by applying ultrasonic pulses at different forward powers, while Figure 4 displays the quantitative results in terms of crystal number and size. The experimentally measured induction time and the theoretically predicted homogeneous nucleation rate are shown in Table 2. This prediction allows the estimation of the required time for growing a nucleus to an observable size $(1.7 \mathrm{~h})$. 




Figure 3. Overview of the lysozyme crystals confined in microdroplets for $\mathrm{S}_{0}=7.2$ : Silent conditions and subjected to pulsed ultrasonic radiation at the indicated forward power $\left(P_{\mathrm{e}}\right)$ and a pulse time duration of $3 \mathrm{~s} \cdot \mathrm{cycle}^{-1}$. [All the pictures were taken $20 \mathrm{~h}$ after the start of each experiment and are representative results from three independent experiments after that time].



Figure 4. Overview of the sonocrystallization experiments for $\mathrm{S}_{0}=7.2$ : Crystal size and number in a single droplet for the applied range of forward power and a pulse time duration of $3 \mathrm{~s}^{\cdot}$ cycle $^{-1}$. [The scale bars are standard deviations from three independent experiments. The dashed lines are indicated to guide the eye.]. 

forward power range.

\begin{tabular}{|c|c|c|c|}
\hline \multirow[t]{2}{*}{$P_{\mathrm{e}}\left[\mathrm{W} \cdot \mathrm{cycle}^{-1}\right]$} & \multicolumn{2}{|c|}{$t_{\text {ind }}[\mathrm{h}]$} & \multirow[t]{2}{*}{$J_{\mathrm{n}}\left[\#\right.$ crystals $\left.\cdot \mu \mathrm{l}^{-1} \cdot \mathrm{s}^{-1}\right]$} \\
\hline & Experimental & Predicted & \\
\hline $\mathbf{0}$ & $3.3 \pm 0.6$ & 3.4 & 0.29 \\
\hline 23 & $2.0 \pm 0.4$ & 1.9 & 1.65 \\
\hline 33 & $1.7 \pm 0.4$ & 1.7 & 42.00 \\
\hline 53 & $1.5 \pm 0.3$ & 1.7 & 41.01 \\
\hline
\end{tabular}

A few number of large crystals are obtained in silent conditions, while the number of crystals increases during the ultrasonic crystallization experiments with the forward power (Figures 3 and 4). However, the results suggest the existence of a critical forward power: Increasing the forward power results in an increase of the nucleation rate until $33 \mathrm{~W} \cdot$ cycle $^{-1}$, while further increase of the forward power no longer leads to any increase of the nucleation rate (Table 2). A possible explanation is that until reaching a certain forward power, the aggregation of protein molecules in solution is favoured, while for further increase there is a competition between aggregate (cluster) formation and breakage. Another possible explanation is based on the depletion zone theory proposed by Heymann and co-workers (Heymann et al., 2014), initially applied to nanolitre-sized droplets. This theory states that when a nucleus is generated, a depletion zone corresponding to the region bordering the growing crystal is formed. The width of this depletion zone defines the number of nucleation events taking place at approximately the same time. Therefore, the width of the depletion zone is a function of the induction time (Heymann et al., 2014). The critical width corresponds to the instant of the last nucleation event. If a cubic shape for this volume is assumed, the number of depletion volumes fitting in the total crystallization volume is 148 . At this point, the critical condition is reached, slightly lower than the number of crystals for the forward powers of $33 \mathrm{~W} \cdot \mathrm{cycle}^{-1}$ and $53 \mathrm{~W} \cdot$ cycle $^{-1}$ (Figure 4). This might indicate that the critical forward power has been achieved.

No observable crystals were formed during the imposed nucleation time, and similar observations were reported by Kakinouchi and co-workers (Kakinouchi et al., 2006). For the detection method used in this study, the measured induction time (Table 2) is higher than $1 \mathrm{~h}$ 
(imposed nucleation time). Additionally, Mao and co-workers (Mao et al., 2020) reported that ultrasound actuation during the crystal growth stage results in smaller crystals, which is possibly due to the simultaneous occurrence of secondary nucleation (and/or breakage) and crystal growth based on the combination between ultrasonic radiation and stirring. The induction time drastically decreases when applying ultrasound radiation, where the effect is already clear for the lowest forward power (Table 2). However, for a clear effect of the ultrasound radiation on the nucleation rate, a higher forward power is required. As explained in the Materials and Methods, the induction time has three contributions: Time to reach a quasi-steady-state distribution of clusters, nucleation time, and growth time of a nuclei until it reaches a detectable size. From the fitting of the induction time along the nucleation rate, it is concluded that it takes around $1.7 \mathrm{~h}$ (Table 2) for a nucleus to grow to an observable size. This might explain the reason for not observing any crystal after reaching the imposed nucleation time.

On the one hand, the increase of the forward power contributes to an increase on the nucleation probability, and hence to decrease the time required to form a nucleus (nucleation time). On the other hand, enhanced nucleation contributes to a faster decay of the lysozyme concentration in the bulk phase. This seems to suggest an intensified effect on the induction time for a forward power of $23 \mathrm{~W} \cdot$ cycle $^{-1}$ in comparison to the crystal number.

\section{Effect of the sonication pulse time on the crystallization outcome}

Without compromising the temperature control, the sonication pulse time was increased from $3 \mathrm{~s} \cdot$ cycle $^{-1}$ to $10 \mathrm{~s} \cdot \mathrm{cycle}^{-1}$. Figure 5 and Table 3 display the obtained results for $\mathrm{S}_{0}=4.2$ in silent conditions and at a forward power of $53 \mathrm{~W} \cdot \mathrm{cycle}^{-1}$. 


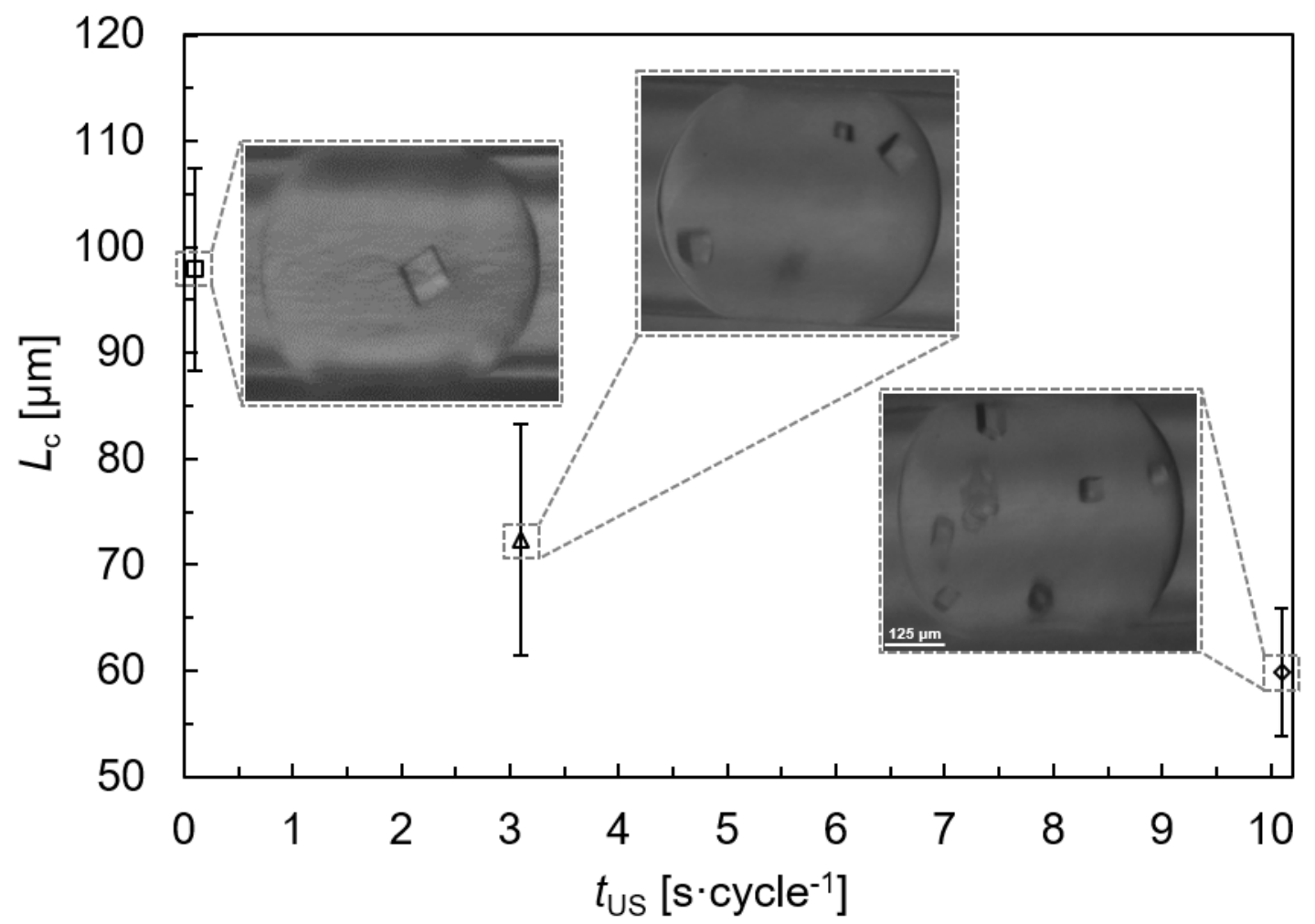

Figure 5. Crystal size (including pictures of the lysozyme crystals confined in the microdroplets) for the crystallization experiments at $\mathrm{S}_{0}=4.2$ in silent conditions and for a forward power of $53 \mathrm{~W} \cdot \mathrm{cycle}^{-1}$. [The scale bars are standard deviations from three independent experiments]. [All the pictures were taken $20 \mathrm{~h}$ after the start of each experiment and are representative results from three independent experiments after that time].

Table 3. Overview of the sonocrystallization experiments for $\mathrm{S}_{0}=4.2$ : Crystal number in a single droplet and predicted nucleation rate for the silent case and different sonication pulse times at $53 \mathrm{~W} \cdot$ cycle $^{-1}$.

\begin{tabular}{ccc}
\hline $\boldsymbol{t}_{\mathrm{US}}\left[\mathbf{s} \cdot \mathbf{c y c l e}^{-1}\right]$ & $\mathbf{N}_{\mathbf{c}}\left[\#\right.$ crystals $\cdot$ droplet $\left.^{-1}\right]$ & $\boldsymbol{J}_{\mathbf{n}}\left[\#\right.$ crystals $\left.^{-1} \boldsymbol{\mu \mathbf { l } ^ { - 1 }} \cdot \mathbf{s}^{-1}\right]$ \\
\hline $\mathbf{0}$ & $0.3 \pm 0.02$ & 0.0014 \\
\hline $\mathbf{3}$ & $2.7 \pm 0.7$ & 0.046 \\
\hline $\mathbf{1 0}$ & $5.6 \pm 1.1$ & 0.15 \\
\hline
\end{tabular}

The droplets subjected to a longer sonication pulse time $\left(10 \mathrm{~s}^{\left.\cdot \mathrm{cycle}^{-1}\right)}\right.$ generated smaller crystals (from $60 \mu \mathrm{m}$ to $72 \mu \mathrm{m}$ ) (Figure 5). However, the main effect comes from the supersaturation as shown in Figures $4\left(\mathrm{~S}_{0}=7.2\right)$ and $5\left(\mathrm{~S}_{0}=4.2\right)$. At identical forward power $\left(53 \mathrm{~W} \cdot \mathrm{cycle}^{-1}\right)$ and sonication pulse time $\left(3 \mathrm{~s} \cdot \mathrm{cycle}^{-1}\right)$, the crystal size is reduced by half with the increased initial supersaturation ratio (from $32 \mu \mathrm{m}$ to $72 \mu \mathrm{m}$ ). This crystal size is further reduced for the droplets exposed to a longer sonication pulse time.

On the contrary, (Mao et al., 2020) reported that crystals obtained at a higher initial supersaturation ratio have a larger size, while the crystal number seems to be similar. The authors' 
proposal highlights the fact that higher protein concentrations can provide more solute for the nucleated crystals to grow into a larger size. This might be a contradiction as if higher supersaturation ratios favour nucleation, many nuclei would be formed, which would consume much of the available protein in solution. The possible explanation is a higher protein concentration decay for higher initial supersaturation ratios, which increases the probability of crystal growth in the metastable zone. For lower protein concentration decays (crystallization points above the metastable zone), there is a higher probability for the occurrence of secondary nucleation. The study reported by (Mao et al., 2020) indicates that sonication improves the mixing process, where the crystals obtained under silent conditions seem to aggregate, while the number of crystals is four orders of magnitude lower compared to the one for continuous sonication. Also, (Crespo et al., 2010) showed a similar tendency of larger crystals for higher supersaturation ratios until a maximum crystal size is achieved. After this stage, the crystal size decreases as the supersaturation ratio increases.

The induction time as a function of the total ultrasound energy for several initial supersaturation ratios, including data from reported cases in the literature, as well as the studied cases, is depicted in Figure 6, while the applied nucleation detection strategy for defining the induction time differs between the studies. Mao and co-workers (Mao et al., 2020) identified the first moment of nucleation by measuring the turbidity. Kakinouchi and co-workers (Kakinouchi et al., 2006) used regular microscopic visualization to observe potential nucleation events at different irradiation points. The indicated values were obtained experimentally and not resulting from any prediction. The only exception is for the studied case at $S_{0}=4.2$, where the growing period of a nuclei to reach a detectable size [Equation (2)] is assumed to be identical to the value achieved at $S_{0}=7.2$, while the nucleation time is predicted based on the crystal number and droplet volume $\left[t_{\mathrm{n}}=\left(V_{\mathrm{d}} / \mathrm{N}_{\mathrm{c}}\right)^{2 / 3} / D_{\mathrm{p}}\right]$ (Heymann et al., 2014). 




Figure 6. Induction time ( $\left.t_{\text {ind }}\right)$ as a function of the total ultrasound energy $\left(E_{\text {tot }}\right)$ for the reported (Kakinouchi et al., 2006; Mao et al., 2020) and studied cases $\left[P_{\mathrm{e}}=271.2|389.2| 625.0 \mathrm{~W} \cdot \mu \mathrm{l}^{-1}\right]$ at the indicated initial supersaturation ratios. Kakinouchi and co-workers (2006) have exposed crystallization volumes of $70 \mu 1$ during distinct irradiation times $\left[P_{\mathrm{e}}=1.4 \mathrm{~W} \cdot \mu \mathrm{l}^{-1}\right]$ at different irradiation points, while Mao and co-workers (2020) simultaneously stirred and sonicated volumes of $30 \mathrm{ml}\left[P_{\mathrm{e}}=0.7 / 2.7 \mathrm{~W} \cdot \mathrm{ml}^{-1}\right]$.

Despite the multiparametric nature of the process (e.g. forward power, frequency, and sonication pulse time), it is possible to observe a decreasing tendency of the induction time for higher energy inputs (Figure 6). As suggested above, there might exist a critical energy level from which further increase no longer introduces any effect on the induction time under ultrasonic crystallization. Moreover, from a certain energy level, similar induction times are reached, regardless of the initial supersaturation ratio.

Although $\mathrm{S}_{0}=7.2$ has $100 \%$ nucleation probability (this point belongs to the nucleation zone), $\mathrm{S}_{0}=4.2$ only has $34 \%$, which is a small margin below the metastability limit (see Materials and Methods, Figure 2). Due to the supersaturation ratios close to the metastability limit, slight changes in the crystallization conditions, can lead to the absence of any nucleation event. This is the case for $\mathrm{S}_{0}=3.8$, where the nucleation probability is zero at both silent and ultrasonic 
conditions after the crystallization time $(20 \mathrm{~h})$. (Crespo et al., 2010) conducted crystallization experiments in a volume approximately two orders of magnitude larger than the studied cases. The results point out a shift in the metastability limit when applying ultrasonic radiation. This seems to indicate that the influence of ultrasound is limited for micro-sized droplets. Thereby, the effect of the ultrasonic radiation on the droplet volume still needs further research as, at our best knowledge, there is not a parametric study predicting the minimum requirements of ultrasonic energy per droplet volume to accelerate the production of the first nucleation centres.

From the reported mechanisms of ultrasonic crystallization, besides cavitation, heterogeneous nucleation and cooling effect might be the ones contributing to the decrease of induction time and crystal size (Kim and Suslick, 2018; Nalajala and Moholkar, 2011; Takano et al., 1997). The forward power increase promotes both mechanisms until a maximum nucleation rate is reached. The resonant cavitation bubble size of $82 \mu \mathrm{m}$ results in a temperature difference on the cavitation bubble surface of $10^{\circ} \mathrm{C}(\mathrm{Hem}, 1967)$. The point corresponding to $\mathrm{S}_{0}=3.8$ $\left(T=25^{\circ} \mathrm{C}\right.$ ) belongs to the metastable zone (Figure 2). For a decrease of $10^{\circ} \mathrm{C}$, this point $\left(\mathrm{S}_{0}=10.8, T=15^{\circ} \mathrm{C}\right)$ could be shifted to the nucleation zone, which would normally trigger nucleation under continuous ultrasound irradiation. Nevertheless, for the studied conditions, it is not possible to decide which mechanism is dominant as the proposed ones activate the interface of the cavitation bubbles. Furthermore, a competition between homogeneous and heterogeneous nucleation should not be neglected. On the one hand, cavitation bubbles might act directly as nucleation sites (gas-liquid interface). Also, liquid-liquid interface might promote nucleation. Following this hypothetic scenario, the crystals could still be uniform, but nucleation would be defined as heterogeneous nucleation. On the other hand, it is still reasonable to assume that the droplet interface is macroscopically homogeneous, so the nuclei formation is spontaneous and, consequently, there is the absence of preferential crystallization active spots (Figure 3). For this last scenario, nucleation would be defined as homogeneous nucleation (Gibbs, 1878). Figure 7 depicts the lysozyme crystal size distribution for silent and ultrasonic crystallization experiments. 


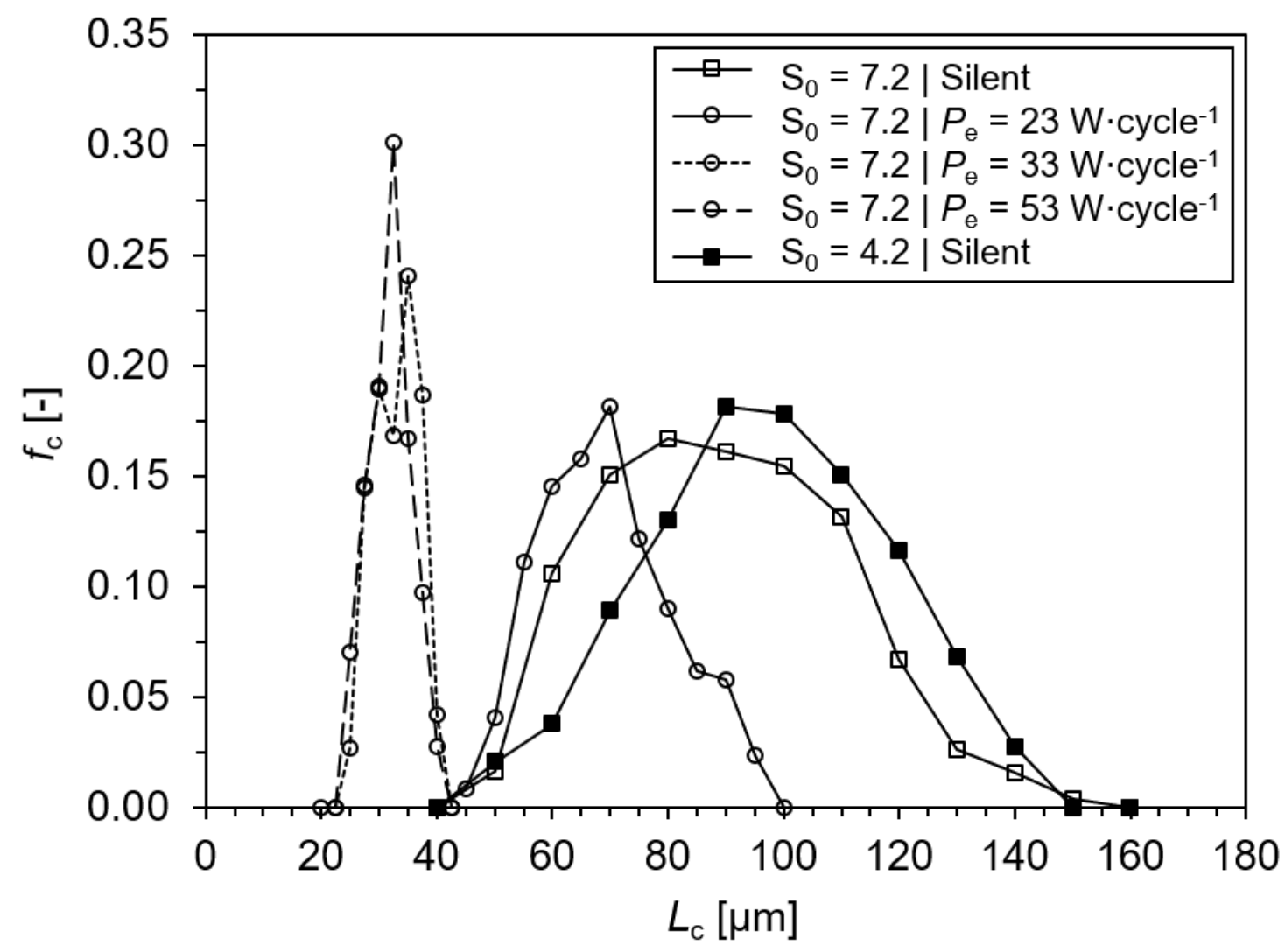

Figure 7. Protein crystal size distribution for silent and ultrasonic crystallization experiments. $\left[f_{\mathrm{c}}\right.$ is the fraction of total droplets that have a specific number of crystals].

The obtained crystals under ultrasonic radiation result in a narrow size distribution, especially for higher forward powers (Figure 7). It seems that aggregation did not occur for both silent and ultrasonic crystallization experiments (Figures 3 and 5). The crystal size uniformity is due to the cavitation effect. Micromixing increase leads to a homogeneous local distribution of supersaturation in the droplets. Thereby, the crystals might have nucleated at the same time, which results in the formation of crystals with similar size. Finally, besides narrower size distribution, crystals are also smaller (Figure 7). The crystals formed at $33 \mathrm{~W} \cdot$ cycle $^{-1}$ and $53 \mathrm{~W} \cdot$ cycle $^{-1}$ present a similar size distribution, which corresponds to reaching the critical region. The distribution becomes broader for the cases under silent conditions. 


\section{Conclusion}

Ultrasonic crystallization promotes nuclei generation during the lysozyme crystallization process in droplets. This contributes to a significant reduction of the induction time and crystal size. Besides the increase of the nucleation rate, the crystal size under ultrasound irradiation is uniform without the indication of protein precipitation. The initial supersaturation ratio has a crucial contribution to the crystal size for silent experiments, while there is a threshold for induction time under ultrasonic crystallization. This is noticed on the small number of larger crystals obtained for all the conditions under lower initial supersaturation ratio $\left(S_{0}=4.2\right)$, while many small crystals are produced for the higher supersaturation ratio $\left(S_{0}=7.2\right)$. The higher nucleation probability by increasing the initial supersaturation ratio results in an increase of the nucleation rate, roughly two orders of magnitude for silent conditions, and three orders of magnitude for a sonication pulse time of $3 \mathrm{~s}^{\cdot} \mathrm{cycle}^{-1}$. The sonication pulse time increase from $3 \mathrm{~s}^{\cdot} \mathrm{cycle}^{-1}$ to $10 \mathrm{~s} \cdot \mathrm{cycle}^{-1}$ results in a threefold nucleation rate increase. Finally, this work provides further perspectives for its extension to hard-to-crystallize biological macromolecules by promoting nucleation using ultrasound without compromising the control of the crystal properties.

\section{Acknowledgements}

S.K. acknowledges funding from the European Research Council under the ERC Starting Grant agreement no. 677169-MicroParticleControl. This work was financially supported by: Base Funding - UIDB/00511/2020 of the Laboratory for Process Engineering, Environment, Biotechnology and Energy - LEPABE - funded by national funds through the FCT/MCTES (PIDDAC).

\section{References}

Alexander, L.F., Radacsi, N., 2019. Application of electric fields for controlling crystallization. CrystEngComm 21, 5014-5031. https://doi.org/10.1039/c9ce00755e

Anna, S.L., 2016. Droplets and Bubbles in Microfluidic Devices. Annual Review of Fluid Mechanics 48, 285-309. https://doi.org/10.1146/annurev-fluid-122414-034425 
Astier, J.P., Veesler, S., Boistelle, R., 1998. Protein crystals orientation in a magnetic field. Acta Crystallographica Section D: Biological Crystallography 54, 703-706. https://doi.org/10.1107/S0907444997020052

B.V., N.I.E., 2019. NIS-Elements Imaging Software [WWW Document]. URL https://www.microscope.healthcare.nikon.com/en_EU/products/software/nis-elements/niselements-advanced-research (accessed 11.1.19).

Cao, H.-L., Yin, D.-C., Guo, Y.-Z., Ma, X.-L., He, J., Guo, W.-H., Xie, X.-Z., Zhou, B.-R., 2012. Rapid crystallization from acoustically levitated droplets. The Journal of the Acoustical Society of America 131,3164-3172. https://doi.org/10.1121/1.3688494

Crespo, R., Martins, P.M., Gales, L., Rocha, F., Damas, A.M., 2010. Potential use of ultrasound to promote protein crystallization. Journal of Applied Crystallography 43, 1419-1425. https://doi.org/10.1107/S0021889810040951

Delacour, C., Lutz, C., Kuhn, S., 2019. Pulsed ultrasound for temperature control and clogging prevention in micro-reactors. Ultrasonics Sonochemistry 55, 67-74. https://doi.org/10.1016/j.ultsonch.2019.03.012

Dombrowski, R.D., Litster, J.D., Wagner, N.J., He, Y., 2010. Modeling the Crystallization of Proteins and Small Organic Molecules in Nanoliter Drops. AIChE Journal 56, 79-91. https://doi.org/10.1002/aic.12001

Dong, Z., Fernandez Rivas, D., Kuhn, S., 2019. Acoustophoretic focusing effects on particle synthesis and clogging in microreactors. Lab on a Chip 19, 316-327. https://doi.org/10.1039/C8LC00675J

Dong, Z., Udepurkar, A.P., Kuhn, S., 2020. Synergistic effects of the alternating application of low and high frequency ultrasound for particle synthesis in microreactors. Ultrasonics Sonochemistry 60, 104800-104809. https://doi.org/10.1016/j.ultsonch.2019.104800

Ducruix, A., Giegé, R., 1999. Crystallization of Nucleic Acids and Proteins: A Practical Approach, $2^{\text {nd }}$ Edition. Oxford University Press.

Durán-Olivencia, M.A., Otálora, F., 2013. A Brownian model for crystal nucleation. Journal of Crystal Growth 380, 247-255. https://doi.org/10.1016/j.jcrysgro.2013.06.035

Eder, R.J.P., Schrank, S., Besenhard, M.O., Roblegg, E., Gruber-Woelfler, H., Khinast, J.G., 2012. Continuous sonocrystallization of acetylsalicylic acid (ASA): Control of crystal size. Crystal Growth \& Design 12, 4733-4738. https://doi.org/10.1021/cg201567y

Ferreira, J., Castro, F., Rocha, F., Kuhn, S., 2018. Protein crystallization in a droplet-based microfluidic device: Hydrodynamic analysis and study of the phase behaviour. Chemical Engineering Science 191, 232-244.

Forsythe, E.L., Judge, R.A., Pusey, M.L., 1999. Tetragonal Chicken Egg White Lysozyme Solubility in Sodium Chloride Solutions. Journal of Chemical \& Engineering Data 44, $637-$ 640. https://doi.org/10.1021/je980316a

Galkin, O., Vekilov, P.G., 1999. Direct Determination of the Nucleation Rates of Protein Crystals. The Journal of Physical: Chemistry B 103, 10965-10971. https://doi.org/10.1021/jp992786x

García-Ruiz, J.M., 2003. Nucleation of protein crystals. Journal of Structural Biology 142, 22 31. https://doi.org/10.1016/S1047-8477(03)00035-2

Gerdts, C.J., Tereshko, V., Yadav, M.K., Dementieva, I., Collart, F., Joachimiak, A., Stevens, R.C., Kuhn, P., Kossiakoff, A., Ismagilov, R.F., 2006. Time-controlled microfluidic seeding in $\mathrm{nL}$-volume droplets to separate nucleation and growth stages of protein crystallization. 
Angewandte Chemie - International Edition 45, 8156-8160.

Gibbs, J.W., 1878. On the equilibrium of heterogeneous substances, American Journal of Science. https://doi.org/10.2475/ajs.s3-16.96.441

Günther, A., Jensen, K.F., 2006. Multiphase microfluidics: from flow characteristics to chemical and materials synthesis. Lab on a Chip 6, 1487-1503. https://doi.org/10.1039/b609851g

Hem, S.L., 1967. The effect of ultrasonic vibrations on crystallization processes. Ultrasonics 5, 202-207. https://doi.org/10.1016/0041-624X(67)90061-3

Heymann, M., Opthalage, A., Wierman, J.L., Akella, S., Szebenyi, D.M.E., Gruner, S.M., Fraden, S., 2014. Room-temperature serial crystallography using a kinetically optimized microfluidic device for protein crystallization and on-chip X-ray diffraction. IUCrJ 1, 349360. https://doi.org/10.1107/S2052252514016960

Howard, E.I., Fernandez, J.M., Garcia-Ruiz, J.M., 2009. On the mixing of protein crystallization cocktails. Crystal Growth \& Design 9, 2707-2712. https://doi.org/10.1021/cg801352d

ImageJ [WWW Document], 2019. . ImageJ. URL https://imagej.net/ImageJ (accessed 11.15.19).

Jiang, M., Papageorgiou, C.D., Waetzig, J., Hardy, A., Langston, M., Braatz, R.D., 2015. Indirect ultrasonication in continuous slug-flow crystallization. Crystal Growth \& Design 15, 24862492. https://doi.org/10.1021/acs.cgd.5b00263

Kakinouchi, K., Adachi, H., Matsumura, H., Inoue, T., Murakami, S., Mori, Y., Koga, Y., Takano, K., Kanaya, S., 2006. Effect of ultrasonic irradiation on protein crystallization. Journal of Crystal Growth 292, 437-440. https://doi.org/10.1016/j.jcrysgro.2006.04.051

Kim, H.N., Suslick, K.S., 2018. The effects of ultrasound on crystals: Sonocrystallization and sonofragmentation. Crystals 8. https://doi.org/10.3390/cryst8070280

Kitayama, H., Yoshimura, Y., So, M., Sakurai, K., Yagi, H., Goto, Y., 2013. A common mechanism underlying amyloid fibrillation and protein crystallization revealed by the effects of ultrasonication. Biochimica \& Biophysica Acta - Proteins and Proteomics 1834, 2640-2646. https://doi.org/10.1016/j.bbapap.2013.09.016

Lau, B.T.C., Baitz, C.A., Dong, X.P., Hansen, C.L., 2007. A Complete Microfluidic Screening Platform for Rational Protein Crystallization. Journal of the American Chemical Society 129, 454-455. https://doi.org/10.1021/ja065855b

Li, F., Lakerveld, R., 2018. Electric-Field-Assisted Protein Crystallization in Continuous Flow. Crystal Growth \& Design 18, 2964-2971. https://doi.org/10.1021/acs.cgd.8b00095

Maeki, M., Teshima, Y., Yoshizuka, S., Yamaguchi, H., Yamashita, K., Miyazaki, M., 2014. Controlling protein crystal nucleation by droplet-based microfluidics. ChemPubSoc Europe 20, 1049-1056. https://doi.org/10.1002/chem.201303270

Mao, Y., Li, F., Wang, T., Cheng, X., Li, G., Li, D., Zhang, X., Hao, H., 2020. Enhancement of Lysozyme Crystallization under Ultrasound Field. Ultrasonics Sonochemistry 63, 1-7. https://doi.org/10.1016/j.ultsonch.2020.104975

Mullin, J.W., 2001. Crystallization, $4^{\text {th }}$ Edition. Butterworth-Heinemann. Oxford. https://doi.org/10.1002/0471238961.0318251918152119.a01.pub3

Nagy, Z.K., Braatz, R.D., 2012. Advances and New Directions in Crystallization Control. Annual Review of Chemical \& Biomolecular Engineering 3, 55-75. https://doi.org/10.1146/annurev-chembioeng-062011-081043

Nalajala, V.S., Moholkar, V.S., 2011. Investigations in the physical mechanism of sonocrystallization. Ultrasonics Sonochemistry $\quad 18, \quad 345-355$. 
https://doi.org/10.1016/j.ultsonch.2010.06.016

Nanev, C.N., Penkova, A., 2001. Nucleation of lysozyme crystals under external electric and ultrasonic fields. Journal of Crystal Growth 232, 285-293. https://doi.org/10.1016/S00220248(01)01169-1

Okutsu, T., 2007. Photochemically-induced crystallization of protein. Journal of Photochemistry and Photobiology C: Photochemistry Reviews 8, 143-155. https://doi.org/10.1016/j.jphotochemrev.2007.06.002

Ratsimba, B., Biscans, B., Delmas, H., Jenck, J., 1999. Sonocrystallization: The end of empiricism? A review on the fundamental investigations and the industrial developments. KONA Powder and Particle Journal 17, 38-48. https://doi.org/10.14356/kona.1999010

Revalor, E., Hammadi, Z., Astier, J.P., Grossier, R., Garcia, E., Hoff, C., Furuta, K., Okustu, T., Morin, R., Veesler, S., 2010. Usual and unusual crystallization from solution. Journal of Crystal Growth 312, 939-946. https://doi.org/10.1016/j.jcrysgro.2010.01.006

Rodríguez-Romero, A., Escofet, N.E., Pareja-Rivera, C., Moreno, A., 2017. Crystal Growth of High-Quality Protein Crystals under the Presence of an Alternant Electric Field in PulseWave Mode, and a Strong Magnetic Field with Radio Frequency Pulses Characterized by X-ray Diffraction. Crystals 7, 1-13. https://doi.org/10.3390/cryst7060179

Rossi, D., Jamshidi, R., Saffari, N., Kuhn, S., Gavriilidis, A., Mazzei, L., 2015. Continuous-Flow Sonocrystallization in Droplet-Based Microfluidics. Crystal Growth \& Design 15, 55195529. https://doi.org/10.1021/acs.cgd.5b01153

Ruecroft, G., Hipkiss, D., Ly, T., Maxted, N., Cains, P.W., 2005. Sonocrystallization: The use of ultrasound for improved industrial crystallization. Organic Process Research \& Development 9, 923-932. https://doi.org/10.1021/op050109x

Seemann, R., Brinkmann, M., Pfohl, T., Herminghaus, S., 2012. Droplet based microfluidics. Reports on Progress in Physics 75, 1-41. https://doi.org/10.1088/0034-4885/75/1/016601

Selimović, S., Gobeaux, F., Fraden, S., 2010. Mapping and manipulating temperatureconcentration phase diagrams using microfluidics. Lab on a Chip 10, 1696-1699. https://doi.org/10.1039/b925661j

Takano, K.J., Harigae, H., Kawamura, Y., Ataka, M., 1997. Effect of hydrostatic pressure on the crystallization of lysozyme based on in situ observations. Journal of Crystal Growth 171, 554-558. https://doi.org/10.1016/S0022-0248(96)00702-6

Taleb, M., Didierjean, C., Jelsch, C., Mangeot, J.P., Capelle, B., Aubry, A., 1999. Crystallization of proteins under an external electric field. Journal of Crystal Growth 200, 575-582. https://doi.org/10.1016/S0022-0248(98)01409-2

Veesler, S., Furuta, K., Horiuchi, H., Hiratsuka, H., Ferté, N., Okutsu, T., 2006. Crystals from light: Photochemically induced nucleation of hen egg-white lysozyme. Crystal Growth \& Design 6, 1631-1635. https://doi.org/10.1021/cg0506424

Yan, E.K., Zhang, C.Y., He, J., Yin, D.C., 2016. An overview of hardware for protein crystallization in a magnetic field. International Journal of Molecular Sciences 17, 1-19. https://doi.org/10.3390/ijms17111906

Yin, D.-C., 2015. Protein crystallization in a magnetic field. Progress in Crystal Growth \& Characterization of Materials 61, 1-26. https://doi.org/10.1016/j.pcrysgrow.2015.03.001

Zhang, Z., Sun, D.W., Zhu, Z., Cheng, L., 2015. Enhancement of Crystallization Processes by Power Ultrasound: Current State-of-the-Art and Research Advances. Comprehensive Reviews in Food Science and Food Safety 14, 303-316. https://doi.org/10.1111/1541- 
4337.12132

Zheng, B., Roach, L.S., Ismagilov, R.F., 2003. Screening of Protein Crystallization Conditions on a Microfluidic Chip Using Nanoliter-Size Droplets. Journal of the American Chemical Society $125,11170-11171$. https://doi.org/10.1021/ja037166v 